\title{
Artificial intelligence on MRI for molecular subtyping of diffuse gliomas: feature comparison, visualization, and correlation between radiomics and deep learning
}

\author{
Zijian Zhou' ${ }^{10}$
}

Received: 1 September 2021 / Revised: 28 September 2021 / Accepted: 12 October 2021 / Published online: 26 November 2021

(c) The Author(s), under exclusive licence to European Society of Radiology 2021

Given advances in tumor molecular analysis, the use of imaging phenotypes to noninvasively determine brain tumor genotypes has become an intriguing possibility [1]. According to the World Health Organization's 2016 revised classification guidelines, diffuse gliomas are classified into five molecular subtypes: (1) oligodendroglioma and anaplastic oligodendroglioma with isocitrate dehydrogenase (IDH) mutation and $1 \mathrm{p} / 19 \mathrm{q}$ codeletion; (2) diffuse and anaplastic astrocytoma with IDH mutation; (3) diffuse astrocytoma with wild-type IDH; (4) glioblastoma (GBM) with IDH mutation; and (5) GBM with wild-type IDH. The first three are low-grade gliomas (LGGs) with grades of 2 or 3, and the latter two are GBM with the grade of 4 . Noninvasively and preoperatively differentiating the molecular subtypes of diffuse gliomas is critical for customizing patient treatment and prognosis prediction. However, completing such tasks can be challenging with conventional statistical methods, as correlations between imaging traits and molecular subtypes can be hard to identify, and discoveries may be inconsistent between studies [2].

Artificial intelligence, especially machine learning and deep learning, has gained a tremendous amount of popularity and has been widely applied in the imaging field. Such data-driven methods provide novel approaches to tackle problems that are challenging to solve with conventional methods. Particularly, when applied in medical images, machine learning is generally referred as conventional radiomics, which requires manually crafted features computed

This comment refers to the article available at https://doi.org/10. 1007/s00330-021-08237-6.

Zijian Zhou

zzhou13@mdanderson.org

1 Department of Imaging Physics, The University of Texas MD Anderson Cancer Center, 1400 Pressler St., Unit 1472, Houston, TX 77030, USA from images for prediction. Such features include firstorder features (for instance pixel intensity mean, median, and histograms), second-order features (for instance texture analysis), and higher order features. In contrast, instead of using manually pre-defined features, deep learning uses 2D or 3D convolutional neural networks (CNNs) to "automatically" extract features from the input images. Therefore, the extracted features, especially from the deeper CNN layers, can be less interpretable. Being able to interpret these features plays a significant role in improving our understanding of deep learning [3].

In their article, Li et al. systematically compared the uses of radiomics and deep learning for the molecular subtyping of diffuse gliomas [4]. Given a large dataset of 780 patients for training and 236 patients for independent testing, as well as multiple MRI sequences, including T1-weighted, T2-weighted, and T1 contrast-enhanced sequences, both radiomics and deep learning achieved outstanding results, with accuracies of around $80 \%$ in differentiating between LGGs and GBM and among LGG subclasses. However, the accuracy was lower-around 70\%-in differentiating among GBM subclasses. The authors also discovered that deep learning consistently achieved better performance than radiomics in most of the binary classification tasks. To compare the extracted imaging features between radiomics and deep learning, the authors conducted a series of studies using feature visualization and correlation.

First, Li et al. used $t$-distributed stochastic neighbor embedding ( $t$-SNE) to visualize the high dimensional features. In general, $t$-SNE is a powerful tool for reducing data dimensionality and provides a more intuitive and straightforward approach to data visualization [5]. It is interesting to note that, under the same settings, features extracted by the CNN were much more clustered and had a clearer pattern than those extracted by radiomics. Such a higher degree of clustering may explain the improved performance of $\mathrm{CNN}$ over radiomics. Besides $t$-SNE, the authors used the Circos 
tool to examine correlations between sparsely selected radiomics features and top-ranked CNN features [6]. It is remarkable that these features had low correlations. Most of the correlations existed in the basic task of glioma grading. For the more detailed task of GBM subtype differentiation, only one pair of features had significant correlation. Such low correlations indicate that CNN may extract drastically different features than radiomics models do.

Besides comparing and correlating the features of radiomics and CNN, the authors also examined the consistency of feature extraction for both approaches using a subset of 180 patients. The tumor regions were re-delineated, and the features were re-computed. Remarkably, for both radiomics and deep learning, the re-computed features were highly correlated to the previously computed ones. However, the CNN features again showed higher intraclass correlation coefficients, indicating that $\mathrm{CNN}$ may be able to extract more stable features than radiomics.

In addition to these notable studies of feature visualization, correlation, and comparison, several limitations of the research need to be discussed. As the authors mentioned, although the study had a huge sample size, the patient number decreased as the classification became finer. However, such a scenario is understandable, and the subtyping results should be robust given that they are based on relatively large testing groups. Another limitation is the tumor imaging volume the authors chose for the CNN. Although the authors used a 2.5D input format with skipped slices, in nature it was still a 2D convolution, whereas radiomics used 3D tumor volume as input. Such dimensional differences may lead to biased feature comparisons and may affect the feature correlations between radiomics and deep learning.

In conclusion, $\mathrm{Li}$ et al. developed radiomics and deep learning models for the molecular subtyping of diffuse gliomas, and the models achieved high performance. They also performed a series of studies comparing, visualizing, and correlating the features of radiomics and deep learning, showing that features extracted from the two approaches can be drastically different. Nonetheless, deep learning features are deemed more clustered and consistent compared to radiomics features. Such studies can help us better understand deep learning and provide more guidance for medical imaging applications of artificial intelligence.

Funding This study has received funding by Siemens Healthineers. The author states that this editorial comment has not received any funding.

\section{Declarations}

Guarantor The scientific guarantor of this publication is Zijian Zhou.

Conflict of interest Zijian Zhou is currently an instructor at the Department of Imaging Physics, The University of Texas MD Anderson Cancer Center. He is partially supported by a research grant from Siemens Healthineers.

Statistics and biometry No complex statistical methods were necessary for this paper.

Informed consent Written informed consent was not required for this study because it is an editorial comment.

Ethical approval Institutional review board approval was obtained. Institutional review board approval was not required because it is an editorial comment.

Methodology

- Editorial comment

\section{References}

1. Diehn M, Nardini C, Wang DS et al (2008) Identification of noninvasive imaging surrogates for brain tumor gene-expression modules. Proc Natl Acad Sci U S A 105:5213-5218

2. Van Meter T, Dumur C, Hafez N, Garrett C, Fillmore H, Broaddus WC (2006) Microarray analysis of MRI-defined tissue samples in glioblastoma reveals differences in regional expression of therapeutic targets. Diagn Mol Pathol 15:195-205

3. Wang CJ, Hamm CA, Savic LJ et al (2019) Deep learning for liver tumor diagnosis part II: convolutional neural network interpretation using radiologic imaging features. Eur Radiol 29:3348-3357

4. Li Y, Wei D, Liu X et al (2021) Molecular subtyping of diffuse gliomas using magnetic resonance imaging: comparison and correlation between radiomics and deep learning. Eur Radiol. https:// doi.org/10.1007/s00330-021-08237-6

5. Belkina AC, Ciccolella CO, Anno R, Halpert R, Spidlen J, SnyderCappione JE (2019) Automated optimized parameters for t-distributed stochastic neighbor embedding improve visualization and analysis of large datasets. Nat Commun 10:1-12

6. Krzywinski M, Schein JE, Birol I et al (2009) Circos: an information aesthetic for comparative genomics. Genome Res 19:1639-1645

Publisher's note Springer Nature remains neutral with regard to jurisdictional claims in published maps and institutional affiliations. 